\title{
La mort des victimes civiles et des combattants dans les discours d'information des journaux télévisés. Le conflit armé en Colombie
}

Yeny Serrano, The Death of Victims and Combatants in Television News. The armed Conflict in Colombia

\section{Yeny Serrano}

\section{(2) OpenEdition \\ Journals}

\section{Édition électronique}

URL : http://journals.openedition.org/questionsdecommunication/2000

DOI : 10.4000/questionsdecommunication.2000

ISSN : 2259-8901

\section{Éditeur}

Presses universitaires de Lorraine

Édition imprimée

Date de publication : 31 décembre 2011

Pagination : 79-104

ISBN : 978-2-8143-0108-5

ISSN : 1633-5961

\section{Référence électronique}

Yeny Serrano, «La mort des victimes civiles et des combattants dans les discours d'information des journaux télévisés. Le conflit armé en Colombie », Questions de communication [En ligne], 20 | 2011, mis en ligne le 01 février 2014, consulté le 01 mai 2019. URL : http://journals.openedition.org/ questionsdecommunication/2000; DOI : 10.4000/questionsdecommunication.2000 


\section{LA MORT DES VICTIMES CIVILES ET DES COMBATTANTS DANS LES DISCOURS D'INFORMATION DES JOURNAUX TÉLÉVISÉS. LE CONFLIT ARMÉ EN COLOMBIE}

Résumé. - Conscients de l'influence que pouvaient avoir leurs discours d'information dans l'évolution et la recrudescence du conflit armé en Colombie, les journalistes colombiens entreprirent dès les années 90 de diffuser moins d'images violentes à la télévision. Ainsi ont-ils décidé de présenter les images des morts et des blessés uniquement en noir et blanc, puis de ne diffuser ces images que dans les cas où cela s'avérerait « strictement nécessaire ». Ces mesures qui, depuis, ont été modifiées ou abandonnées, visaient à ne pas rendre les téléspectateurs insensibles en diffusant des images violentes de manière récurrente. Deux décennies plus tard, cet article s'interroge sur la place de la mort dans les discours d'information médiatique concernant le conflit armé et sur la manière d'informer de la mort des civils et des combattants. Sur la base d'un corpus télévisuel extrait des quatre journaux télévisés nationaux du pays, entre juin 2006 et juin 2008, il s'agit d'analyser la manière dont l'exhibition ou l'occultation de la mort associée à la confrontation armée en Colombie, ainsi que la manière de la désigner, répond à des enjeux stratégiques des acteurs en conflit qui cherchent à légitimer leur guerre en discréditant l'adversaire à travers les médias de masse.

Mots clés. - Mort, combattants, civils, légitimité, conflit armé, Colombie. 
A xé sur les enjeux de la re-présentation' de la mort dans les médias d'information, cet article aborde la mort en contexte de guerre, autrement dit la mort violente, non naturelle et qui n'est pas liée à la fin « normale » d'un parcours de vie. Dans une guerre, « on ne meurt pas ; on est tué ou on se laisse mourir » (Thomas, 2003 : I I ) pour défendre une cause politique, religieuse, identitaire, patriotique, etc. Même si, d'un point de vue anthropologique, la guerre peut être considérée comme une pratique nécessaire pour exercer les pulsions agressives de l'homme ou comme régulateur démographique (ibid. : II-12), la mort intrinsèque à la guerre transgresse les normes sociales et sa gestion constitue un enjeu dans la stratégie militaire des armées (Mercier, 2004). Dans le cadre des opérations psychologiques qui font partie de toute stratégie militaire, les acteurs armés minimisent et justifient la mort causée par leurs troupes et instrumentalisent la mort causée par l'adversaire (Géré, 1997). Exhiber les morts ennemis permet de signifier à l'adversaire l'ampleur de sa défaite et de signaler à sa propre population que la victoire est proche. Montrer ses propres morts peut servir à convaincre la population civile et/ou à renforcer son positionnement contre l'ennemi. Toutefois, cette « monstration » des morts peut, en même temps, démoraliser les troupes et consolider l'idée de la défaite (Mercier, 2004 : |5|). En d'autres termes, la «monstration » et l'occultation ${ }^{2}$ de la mort font partie intégrante des stratégies des armées dans la justification de la violence et la stigmatisation de l'adversaire (Arboit, 2006).

En nous penchant sur le conflit armé en Colombie $^{3}$ et sa couverture informationnelle par les journaux télévisés nationaux (JT), nous circonscrivons notre champ d'analyse à la re-présentation de la mort de civils et de combattants dans les informations télévisées. Des études comme celles de Frédéric Antoine (1993 : 46) avaient déjà mis en évidence qu'« à la télévision [...], la mort s'est mis à envahir les espaces réservés à l'information ». D'autres concluaient que les médias d'information, dominés par la logique d'émotion, ne s'intéressent qu'à la mort qui résulte des événements extraordinaires (Le Guay, 2008 : 120). À partir de ces constats et sachant que les journalistes colombiens ont décidé de diffuser

\footnotetext{
En répondant à la problématique posée par ces deux dossiers consacrés aux modalités et aux enjeux de la re-présentation de la mort dans les médias d'information, cet article s'inscrit dans la continuité de l'analyse d'A. Rabatel et M.-L. Florea (20II) pour qui la re-présentation est plus qu'une représentation fidèle d'une réalité préexistante.

2 Plus qu'occulter la mort, pour A. Mercier (1993:2), les armées ont mis en place un système de censure consistant à retravailler la manière de présenter la mort aux médias en l'euphémisant et en l'esthétisant.

${ }^{3} \mathrm{Si}$ en Europe, la situation de violence sociopolitique qui règne en Colombie peut être perçue comme une " guerre civile », cette dénomination s'avère délicate à l'intérieur du pays. Après les Protocoles de Genève (1949), l'onv et d'autres organisations internationales, comme le CICR traitent la situation dans ce pays comme un « conflit armé non international » ou un « conflit armé interne ». En droit international, ces deux expressions s'appliquent à ce qu'on désignait comme une « guerre civile » avant 1949. En Colombie, les expressions « conflit », « conflit armé », voire « guerre » se sont imposées dans les discours politiques, médiatiques et académiques. II est très rare que les Colombien-ne-s parlent de « guerre civile ».
} 
moins d'images de morts pour protéger les téléspectateurs, il paraît pertinent de soulever certaines interrogations : quelle place prend la mort dans les discours à visée informative concernant le conflit armé en Colombie ? Comment et pourquoi la mort est-elle dite/montrée dans les informations télévisées se rapportant au conflit armé ? Pour répondre à ces questions, l'article examine la manière dont la mort des civils ${ }^{4}$ et des combattants ${ }^{5}$ (étatiques ${ }^{6}$, paraétatiques ${ }^{7}$ et contre-étatiques ${ }^{8}$ ) est désignée dans les informations télévisées étant donné que la télévision est le média le plus utilisé en Colombie (CNTV, 2009).

Plus concrètement, nous avons analysé les jT nationaux diffusés en semaine le soir : Telepaís et $C M \&$, diffusés par la chaîne publique Canal Uno' ${ }^{9}$ Noticias Caracol et Noticias RCN émis par les deux chaînes privées nationales du pays. Le corpus a été constitué sur la base des émissions diffusées du 12 au 15 juin 2006, du II au 15 décembre 2006, du 25 au 29 juin 2007, du 10 au 14 décembre 2007 et du 16 au 20 juin 2008. Ces dates ont été choisies de façon aléatoire sachant que le conflit est traité quotidiennement par les médias nationaux et que, en Colombie, les archives des médias ne sont pas de libre accès. Au total, le corpus de travail

4 Par « civils », nous faisons référence à la population non armée, rurale ou urbaine. Dans les analyses, nous distinguons les fonctionnaires de l'État, les membres du gouvernement et les autres civils qui ne font pas partie de la vie politique du pays (témoins, victimes, membres d'organisations sociales, avocats ou autres professionnels).

${ }^{5}$ Les lecteurs souhaitant approfondir l'histoire des groupes armés en Colombie et leurs enjeux peuvent se référer, entre autres travaux à ceux de D. Bushnell (1993), D. Garibay (2003), O. Gonzalez (2010) et D. Pécaut (2006).

6 Les Forces armées de l'État (FF.MM. selon l'abréviation utilisée en Colombie) sont constituées de l'Armée, la Police, l'Armée de l'air, la Marine et les services de renseignement. En tant que représentants de l'État, ces groupes revendiquent leur légalité et combattent les groupes armés hors-la-loi : guérillas, paramilitaires et groupes de délinquants sans caractère politique. Pourtant, plusieurs membres des FF.MM. font l'objet d'enquêtes pénales pour avoir collaboré avec les groupes paramilitaires.

7 Les groupes paramilitaires se présentent comme les défenseurs de la classe moyenne et aisée qui, selon eux, sont délaissées par l'État, les laissant à la merci des guérillas. Ils ont été créés pour réaliser le « sale boulot » pour les forces armées de l'État. II consiste à dissuader ou tuer les personnes suspectées de faire partie ou de sympathiser avec les guérillas. Ainsi certaines personnes considèrent-elles les paramilitaires comme un « mal nécessaire » pour combattre les guérillas (PNUD, 2003 : 428 et 437). Entre 2003 et 2006, ces groupes ont été massivement démobilisés dans un processus de remise d'armes, très critiqué, mis en place par le gouvernement du président Álvaro Uribe (2002-2006 et 2006-20 I0). Depuis ces démobilisations, les autorités colombiennes désignent les groupes paramilitaires encore actifs comme des « bandes criminelles ». Cette désignation répond à une double stratégie, d'une part, celle d'affirmer que les paramilitaires n'existent plus et d'autre part, celle de refuser de reconnaitre les motivations politiques des groupes armés hors-la-loi.

${ }^{8}$ Les groupes de guérillas sont essentiellement les FARC et ELN. Dans leurs discours, ceux-ci plaident pour la mise en place d'un État garantissant une meilleure distribution des richesses. Le discours officiel dominant affirme que si les guérillas défendaient des intérêts nobles et légitimes à l'origine, elles sont devenues des «terroristes » dédiées au trafic de drogue. II convient de préciser que le lien entre guérilla et narcotrafic a été conçu comme une stratégie de l'ambassadeur des États-Unis à Bogota en 1984, L. Tambs, dans le but de transformer la lutte anti-drogue en lutte anti guérilla (Pécaut, $2003: 52$ ).

9 En Colombie, la programmation des chaînes publiques n'est pas assurée par l'État. Ce dernier loue des espaces de diffusion à de petites et moyennes entreprises de programmation. 
contient 452 unités d'information ${ }^{10}$ se rapportant au conflit armé. Le souscorpus utilisé dans cette analyse rassemble les 187 unités d'information dans lesquelles la mort d'un civil ou d'un combattant est mentionnée. Une analyse de contenu (Bardin, 1977) assistée par ordinateur (logiciel Atlas.TI, version 5.2) a été menée. De manière à mieux saisir et appréhender les enjeux que revêtent les différentes manières de nommer la mort, les termes avec lesquels les sources et les journalistes désignent la mort ont été recensés en distinguant deux référents : les civils et les combattants.

\section{Considérations préliminaires}

Dans les médias, la question de la désignation est directement liée à celle des sources, autrement dit aux acteurs sociaux à qui les journalistes donnent la parole pour décrire et expliquer les événements dont ils ont été les témoins ou auxquels ils ont directement participé. Ainsi les sources jouent-elles un rôle dans la définition de l'agenda médiatique et dans le cadrage avec lequel les événements sont rapportés par les médias. Dans le cadre de cette analyse, plus de la moitié de 187 informations étudiées ont été élaborées par les journalistes à partir de la version fournie par des sources officielles : $27 \%$ des unités d'information citent ou donnent la parole aux membres des forces armées de l'État (FF.MM.), 16 \% aux fonctionnaires de l'État et $12 \%$ aux membres du gouvernement. En d'autres termes, il est probable que les JT relaient la version du conflit que défendent l'État et les acteurs armés qui le représentent plutôt que celle de l'opposition armée et non armée. Pour ces acteurs étatiques, cette démarche de légitimation de leur violence comporte une double finalité, d'une part, maintenir le moral des troupes et, d'autre part, convaincre la population civile de la légitimité du conflit. Pour parvenir à cette fin, les FF.MM. s'imposent comme les seules sources légitimes et demandent aux journalistes leur inconditionnalité (PAN, 2004). Dans le même dessein, la création de la Commission nationale de télévision par l'État colombien était vouée à exercer une contrainte sur les médias pour qu'ils ne diffusent pas les messages et communiqués des guérillas (accord 0 17, art. IV, CNTV, 1997). De plus, un groupe de propriétaires de médias estime que leur devoir est de soutenir l'État, considéré comme le seul acteur légal et donc légitime du conflit ; ils exercent ainsi une pression sur leurs employés (journalistes) afin qu'ils se conforment au choix et à l'utilisation de certaines sources d'information. Un journaliste affirmait, sous couvert d'anonymat que le directeur du JT pour lequel il travaillait exigeait que des voix officielles soient présentes dans plus des $90 \%$ des informations (MPP. 2006 : 96).

10 Une unité d'information commence avec le lancement fait par les présentateurs, suivi (ou non, dans le cas des brèves) d'un reportage avec des images tournées sur le terrain ou des images d'archives. Le JT indique à ses téléspectateurs la fin d'une information en retournant sur le plateau avec les présentateurs ou avec une coupe de plan en volet - raccord dans lequel l'image disparaît par un bord du cadre pendant que la nouvelle image arrive sur l'écran (Viallon, 1996 : 79). 
Si un groupe de propriétaires et de journalistes estiment ces restrictions légitimes, pour d'autres, elles vont à l'encontre de leur code déontologique. En conséquence, une tension peut prévaloir entre les engagements démocratiques des journalistes colombiens et l'instrumentalisation des médias par les sources journalistiques, ce qui illustre une des particularités qui subsiste dans la couverture médiatique des conflits internes. Dans le cas des guerres étrangères, les journalistes peuvent être moins concernés personnellement. Ils peuvent « plus facilement » afficher une position distanciée à l'égard des parties du conflit. En revanche, dans le cas des conflits internes traités par les médias de ces mêmes pays, les journalistes sont censés mieux connaître la situation. Ils peuvent se sentir plus ou moins impliqués pour défendre ou dénoncer l'un ou l'autre des belligérants et de ce fait reprendre plus ou moins facilement leur lexique de guerre. À cela s'ajoutent les conditions de travail précaires et instables, les pressions exercées sur les journalistes par les propriétaires des médias" et par les membres de groupes armés (Giraldo, Roldán, Flórez, 2003). En effet, l'information qui est portée à la connaissance des citoyen-ne-s représente une arme pour les acteurs en conflit. Ainsi ont-ils intérêt à instrumentaliser les relations qu'ils entretiennent avec les journalistes et les propriétaires des médias dans le but de faire diffuser une version du conflit favorable à leur cause et qui discrédite celle de l'adversaire (Géré, 1997).

Parallèlement, couvrir le conflit armé représente pour les journalistes colombiens un moyen de gagner leur vie en exerçant un métier auquel ils attribuent une fonction sociale importante (Rincón, Ruíz, 2002). Dans leur code déontologique, leurs manuels de rédaction et les formations continues qu'ils organisent, les journalistes revendiquent l'exercice d'un journalisme pour la paix (MPP, ibid.). Ils refusent de prendre parti explicitement en faveur de l'un des acteurs en conflit. Toutefois, ils ne sont pas toujours préparés pour répondre aux contraintes imposées par les propriétaires des médias et par les acteurs en conflit. Ils considèrent que le caractère légal d'un groupe est un critère à prendre en considération et qu'il est légitime de défendre ceux qui, à leur avis, souffrent le plus dans le conflit : les civils. Cette attitude les conduit à assumer un parti pris implicite qui consiste à se positionner contre ceux qui sont responsables de la situation de ces victimes. Les enquêtes menées auprès des journalistes (Giraldo, Roldán, Flórez, 2003 ; MPP. 2006, PAN, 2004 ; Rincón, Ruíz, 2002) ont mis en évidence que, pour faire face à la tension entre engagements démocratiques et pressions, les journalistes ont développé certains « mythes » : ils préfèrent avoir recours aux sources officielles parce qu'elles peuvent répondre publiquement de ce qu'elles disent; ils estiment également que les gens ordinaires sont plus fiables en tant que sources, tout comme l'Église, car ils n'auraient pas d'intérêts

\footnotetext{
"Certains membres des familles qui possèdent les plus importants médias du pays ont fait ou font partie du gouvernement. Par exemple, l'actuel président de la République, J. M. Santos (ancien ministre de la Défense) et l'ancien vice-président, Fr. Santos, appartiennent tous deux à la famille Santos, propriétaire du quotidien national de référence, El Tiempo.
} 
particuliers à défendre. En outre, la genèse du conflit ${ }^{12}$ et son évolution au cours des sept dernières décennies ${ }^{13}$ ont engendré une situation dans laquelle le gouvernement a choisi de défendre l'issue militaire du conflit. Dans cette optique, il a doublé en cinq ans le nombre d'effectifs des FF.MM. (I 47000 en 2003, 329 I3| en 2008) avec le soutien financier et militaire des États-Unis et renforcé le nombre d'opérations militaires (IISS, 2009). Dans ce sens, il apparaît plus judicieux pour le gouvernement de légitimer, notamment à travers les médias de masse, la guerre et l'augmentation du budget consacré à celle-ci, au détriment d'autres secteurs comme la santé ou l'éducation. D'ailleurs, des analyses menées au cours des dix dernières années (López, 2005 ; PAN, 2004) constatent une polarisation de la société (incluant les propriétaires de grands médias et certains journalistes) en faveur de l'issue militaire voulue par le gouvernement ${ }^{14}$.

En d'autres termes, la représentation de la mort liée au conflit armé en Colombie ne dépend pas exclusivement des contraintes médiatiques (de rentabilité et de crédibilité) ou journalistiques (relation avec les sources, exigences de produire des discours distanciés en respectant la ligne éditoriale et le format du média) (Charaudeau, 2005). Elle doit être aussi analysée sous l'angle des pratiques de communication de guerre des acteurs armés, notamment de ceux qui peuvent s'exprimer à travers les médias de masse. La représentation de la mort, parmi d'autres sujets en rapport à la guerre, est soumise à des visées argumentatives des acteurs armés et des responsables politiques - qui dépassent la dimension informative prônée par les JT. Dans cette perspective, il s'agit à présent d'examiner dans quelle mesure la mort liée au conflit armé est présente dans les JT et d'analyser les enjeux portés par les différentes manières de la désigner.

\section{La mort associée au conflit armé dans les informations télévisées}

Au cours des cinq semaines d'information prises en compte dans le cadre de cette analyse, entre juin 2006 et juin 2008, la mort d'un civil ou d'un combattant

\footnotetext{
12 Malgré les controverses relatives à ce sujet, il est possible d'affirmer que les origines de la confrontation armée remontent à la fin des années 40 . Nous tenons à préciser que l'histoire de la confrontation et des groupes en conflit fait l'objet des débats entre des chercheurs inscrits dans des approches qui s'opposent radicalement (voir à ce propos Y. Serrano, 20I0).

13 Cette évolution a été marquée par l'apparition de divers groupes de guérillas et de paramilitaires, l'augmentation de la confrontation entre ces groupes, le déplacement de la population civile et plus récemment, l'échec des dernières négociations du gouvernement avec les guérillas des FARC (19992002) et de l'ELN (2002-2007), ainsi que par la démobilisation massive de groupes paramilitaires entre 2003 et 2006.

${ }_{14}$ Pour une meilleure compréhension des liens entre gouvernement, journalistes, acteurs en conflit et propriétaires des médias, des études sur le terrain en sociologie du journalisme s'avèrent nécessaires pour compléter cette analyse qui s'inscrit dans une approche d'analyse de contenu du produit médiatique d'information.
} 
figurait dans $42 \%$ des 452 unités recensées. Parmi ces informations, plus de la moitié ( 109 sur 187) correspond à la mort de civils. En juin 2007, les FARC ont annoncé la mort en captivitél5 de I I députés régionaux que ce groupe armé avait « privés de liberté $\rangle^{16}$. Pendant deux jours, les JT ont presque exclusivement consacré leurs émissions à ce sujet. En conséquence, dans le corpus retenu, la mort des députés, et donc des civils, est largement surreprésentée par rapport à celle des combattants, alors qu'entre juin 2006 et juin 2008, le nombre de combattants morts (entre 1500 et 2200, Granada, Restrepo, Vargas, 2009 : 39) à cause du conflit armé s'avérait plus élevé que le nombre de civils morts (entre 1000 et 1300, Granada, Restrepo, Vargas, 2009 : 39). Ces résultats suggèrent que les JT ont surmédiatisé la mort des civils ${ }^{17}$ : des 246 mentions faites aux personnes décédées, $66 \%$ concernent des civils et seulement $18 \%$ des combattants (graphique I). De ce fait, le corpus n'est pas représentatif en raison du nombre d'unités d'information qui, pendant deux jours, traitent l'affaire des députés. Nonobstant, cette affaire illustre les enjeux que représente pour les médias et les acteurs du conflit l'instrumentalisation de la mort des civils.

Graphique I : Quels sont les morts mis en évidence par les JT ?18

15 Afin d'exercer des pressions et de démontrer leur capacité militaire, depuis 1996, les FARC ont pris en otage des centaines de militaires, policiers et politicien-ne-s. Une grande partie de ces personnes a été libérée unilatéralement par la guérilla ou, de force, par l'armée colombienne. Parmi ces personnes, 12 députés régionaux avaient été « privés de liberté » en avril 2002. Le 28 juin 2007, les FARC ont envoyé un communiqué de presse pour annoncer la mort en captivité, une dizaine de jours auparavant, de II de ces 12 députés « au milieu d'un feu croisé lorsqu'un groupe militaire non identifié a attaqué le campement où ils se trouvaient » (diffusé par Telepaís, Noticias Caracol et Noticias RCN, le 28/06/07).

16 La manière de nommer les personnalités politiques et les membres des FF.MM. « privés de liberté » par la guérilla pose problème. Selon la dénomination employée (« kidnappé », « retenu », « prisonnier de guerre »), la légitimité accordée à la guérilla n'est pas la même. Nous utilisons l'expression « privé de liberté » employée dans le Protocole additionnel aux Conventions de Genève relatif à la protection des victimes des conflits armés non internationaux. Pour une analyse plus approfondie sur cette question voirY. Serrano (2010).

17 Pour établir si les JT surmédiatisent la mort des civils, il serait nécessaire d'élargir cette analyse avec un corpus exhaustif sur les deux années prises en compte.

18 Le graphique montre la proportion de morts mentionnés sur les 246 mentions totales repérées dans les 187 unités d'information analysées. 
Ces enjeux se manifestent dans les expressions utilisées par les journalistes et les sources pour se référer à la mort de civils et de combattants. Les différents syntagmes recensés dans cette analyse diffèrent quant à l'accent mis sur la violence produite par chaque acteur armé et par rapport au degré d'« humanité » accordé aux morts dont les JT parlent. Selon la désignation employée, les énonciateurs peuvent orienter leurs énoncés en faveur de l'un ou l'autre des belligérants (Veniard, 2003 : 23), ce qui s'avère particulièrement important pour les acteurs armés dans le cadre d'un conflit armé interne puisque la question de la légitimité auprès de la société est centrale : il faut vilipender l'ennemi pour justifier la violence exercée à son égard et gagner le soutien de la population (Géré, 1997). Ainsi les 463 expressions utilisées pour désigner la mort ont-elles été extraites du corpus et analysées dans une perspective communicationnelle de façon à mettre en exergue la manière dont elles contribuent à (dé)légitimer certains acteurs armés. Pour cela, une typologie (présentée dans le tableau I) est suggérée. Elle classe les désignations de la mort selon l'orientation plus ou moins accusatrice à l'égard du responsable de la mort. Les huit catégories proposées permettent d'observer si les énonciateurs désignent la mort avec des euphémismes qui mettent à distance la mort liée au conflit armé (« la mort », 《la mort "affaire"», 《la mort judiciaire », 《la mort indirecte ») ou avec des expressions mettant en exergue la violence (« la mort reprouvée », « la mort évaluée », 《la mort déshumanisée »). Pour construire cette typologie, nous avons pris en considération deux constats mis en évidence par des recherches menées préalablement au sujet de la mort dans les médias. Le premier concerne le tabou autour du cadavre et de la représentation de la mort en Occident (Bertherat, 2003), qui expliquerait, en partie, l'euphémisation de celle-ci ainsi que les mesures prises par les journalistes colombien-ne-s de ne pas montrer de manière récurrente des images de morts et de blessés. Le second concerne la construction des États dans les sociétés occidentales qui a entraîné un processus de domestication de la violence ayant abouti à l'octroi du monopole légitime de la violence physique à l'État (Mercier, 1993 : 3). Ceci expliquerait les exigences faites aux journalistes en Colombie par le gouvernement et ses forces armées de ne pas traiter de la même manière des acteurs armés légaux et illégaux. 


\begin{tabular}{|c|c|c|}
\hline Catégorie & Définition & Lexique concerné19 $^{19}$ \\
\hline « La mort » & $\begin{array}{l}\text { Termes qui pourraient désigner } \\
\text { la mort survenue de manière } \\
\text { naturelle. Ils n'impliquent } \\
\text { pas l'accusation explicite du } \\
\text { responsable et ne mettent pas } \\
\text { l'accent sur la violence. }\end{array}$ & $\begin{array}{l}\text { La muerte (la mort), morir } \\
\text { (mourir), fallecer (décéder), }\end{array}$ \\
\hline La mort « affaire » & $\begin{array}{l}\text { Toponymes désignant des } \\
\text { massacres en fonction de l'endroit } \\
\text { où ils ont eu lieu. }\end{array}$ & Caso Jamundí (affaire Jamundi) \\
\hline La mort judiciaire & $\begin{array}{l}\text { La mort est nommée en utilisant } \\
\text { un registre juridique. }\end{array}$ & $\begin{array}{l}\text { Homicidio (homicide), homicido } \\
\text { agravado (homicide aggravé), } \\
\text { crimen de lesa humanidad (crime } \\
\text { de lèse humanité), crimen de } \\
\text { guerra (crime de guerre) }\end{array}$ \\
\hline La mort suicide & $\begin{array}{l}\text { Termes faisant référence au fait de } \\
\text { se donner la mort. }\end{array}$ & $\begin{array}{l}\text { Suicidarse (se suicider), ahorcarse } \\
\text { (se pendre), quitarse la vida (s'ôter } \\
\text { ou s'enlever la vie) }\end{array}$ \\
\hline La mort réprouvée & $\begin{array}{l}\text { Noms et verbes qui mettent } \\
\text { l'accent sur la violence et à travers } \\
\text { lesquels la mort est fustigée. }\end{array}$ & $\begin{array}{l}\text { Asesinato (assassinat/meurtre), } \\
\text { masacre (massacre), degollar } \\
\text { (égorger), crimen (crime), matar } \\
\text { (tuer), ejecutar (exécuter), aniquilar } \\
\text { (exterminer) }\end{array}$ \\
\hline La mort évaluée & $\begin{array}{l}\text { À la différence de la catégorie } \\
\text { précédente }^{20} \text {, celle-ci regroupe les } \\
\text { expressions dans lesquelles des } \\
\text { adjectifs ou des périphrases sont } \\
\text { utilisées pour critiquer/dénoncer } \\
\text { la mort. }\end{array}$ & $\begin{array}{l}\text { Trágica muerte (mort tragique), } \\
\text { hecho/crimen atroz/horrible (fait/ } \\
\text { crime atroce/horrible), la mayor } \\
\text { masacre (le plus grand massacre), } \\
\text { vil asesinato (vil assassinat), acto } \\
\text { criminal/execrable/infame (acte } \\
\text { criminel/exécrable/infâme), morir } \\
\text { como un perro (mourir comme un } \\
\text { chien), matanza (tuerie/abattage), } \\
\text { crimen imperdonable (crime } \\
\text { impardonnable), }\end{array}$ \\
\hline $\begin{array}{l}\text { La mort } \\
\text { déshumanisée }\end{array}$ & $\begin{array}{l}\text { Lexique utilisé dans un registre } \\
\text { militaire pour nommer la mort } \\
\text { de l'adversaire et qui rappelle } \\
\text { les expressions employées pour } \\
\text { parler de la mise à mort des } \\
\text { animaux. }\end{array}$ & $\begin{array}{l}\text { Dar de baja (liquider), abatir } \\
\text { (abattre) }\end{array}$ \\
\hline
\end{tabular}




\begin{tabular}{|l|l|l|}
\hline \multirow{1}{*}{ La mort indirecte } & $\begin{array}{l}\text { Expressions qui font référence à la } \\
\text { mort en parlant des événements } \\
\text { qui lui sont associés ou de l'absence } \\
\text { de vie. }\end{array}$ & $\begin{array}{l}\text { Velorio (veillée funèbre), entierro } \\
\text { (enterrement), fosas comunes } \\
\text { (fosses communes), por la memoria } \\
\text { de... (à la mémoire de...), sepelio } \\
\text { (obsèques), vivir feliz allá en el cielo } \\
\text { (vivre heureux au ciel), luto (deuil), } \\
\text { su partida (son/leur départ), diosito } \\
\text { se los quisollevar (Dieu a voulu les } \\
\text { prendre), vidas que se perdieron } \\
\text { (des vies qui se sont perdues), } \\
\text { desaparición (disparition), el dolor de } \\
\text { saber que ya no volverá (la douleur } \\
\text { de savoir qu'il ne reviendra pas), } \\
\text { cuatro tablas de madera (quatre } \\
\text { plaques en bois), las viudas (les } \\
\text { veuves), el infortunado hecho (le } \\
\text { fait infortuné), investigación forense } \\
\text { (enquête du médecin légiste) }\end{array}$ \\
\hline
\end{tabular}

Tableau I : Typologie du lexique employé pour désigner la mort.

Plus spécifiquement, le discours des journalistes met en évidence différentes attitudes énonciatives qui vont de l'appropriation du lexique employé par les sources ${ }^{21}$ jusqu'à la distanciation totale vis-à-vis de ce dernier ${ }^{22}$. Entre l'appropriation du lexique et la distanciation à l'égard de celui-ci, on observe un positionnement discursif intermédiaire lorsque les journalistes énoncent les désignations en citant leurs sources (discours rapporté indirect ou narrativisé). Dans le cas de notre objet d'étude, la distanciation énonciative (qui fait partie des règles professionnelles acceptées par les journalistes colombiens) est moindre lorsque les professionnels de l'information se réfèrent à la mort des membres des forces armées de l'État : $70 \%$ des désignations sont assumées par les journalistes alors que seulement $9 \%$ sont prises en charge directement par les sources. La situation est semblable lorsque les journalistes désignent la mort des paramilitaires. En revanche, les deux tiers des expressions utilisées

\footnotetext{
19 Texte traduit de l'espagnol par nous-même.

20 Dans leurs manuels de rédaction et leur code déontologique, les journalistes colombiens préfèrent l'utilisation d'un vocabulaire strictement descriptif; ; 'utilisation d'adjectifs qualificatifs est plutôt défendue. Dès lors, il semblait pertinent de proposer deux catégories pour classer les désignations qui condamnent la mort à travers des noms et des verbes et celles qui le font en utilisant des qualificatifs (adjectifs, périphrases, etc.). Cette façon de procéder a permis de mettre en évidence que le deuxième cas de figure se présente uniquement lorsqu'il s'agit de désigner la mort des civils.

${ }^{21}$ Le cas de l'appropriation vis-à-vis des sources correspond aux expressions énoncées par les journalistes sans citer leurs sources.

22 Le cas de la distanciation vis-à-vis des sources correspond aux expressions énoncées directement par les sources. « Lorsque le jt montre un énonciateur externe à l'écran, l'image correspond à une marque iconique et sonore du discours rapporté direct, dans la mesure où le média audiovisuel permet la prise en charge par le son et/ou l'image de certains aspects indispensables du discours direct 》 (von Münchow, 2004 : 102-103).
} 
pour désigner la mort des civils et des guérilléros correspondent à du discours rapporté direct ou indirect avec lequel ces professionnels cherchent à mettre en scène un éthos d'objectivité.

Quant aux modes de désignation le plus souvent utilisées par les sources (discours direct), plus de la moitié de celles qui ont été classées dans la catégorie « la mort juridique » ont été énoncées par des représentants des institutions étatiques, notamment du pouvoir judiciaire. « La mort déshumanisée » correspond à une catégorie dont la moitié des désignations provient de sources militaires et de représentants du gouvernement (président de la République et ministre de la Défense). En effet, la deshumanisation de l'adversaire constitue le plus souvent une pratique propre aux armées. Enfin, un tiers des désignations se référant à la mort de manière indirecte a été énoncé par les proches des députés morts en captivité. Étant donné que nous n'avons pas constaté de différences significatives entre les quatre JT analysés, les résultats sont présentés de manière groupée -sans différencier chaque chaîne et chaque JT.

\section{La mort des victimes civiles dans les informations télévisées}

Si l'on compare la manière dont les JT traitent la mort des civils et celle des combattants, il est possible de constater que le traitement que les médias d'information accordent à la mort des civils est plus « humain » que celui donné à la mort des combattants, dans la mesure où ils font ressortir des aspects émotionnels. Ainsi une mort distante est-elle présentée de manière à créer une proximité en sensibilisant l'attention des téléspectateurs. Ces morts civils sont donc appréhendés comme la mort d'un proche, ou selon les termes de Vladimir Jankélévitch (1977 : 24-35), comme une mort en deuxième personne. Par ailleurs, et comme cela a été mentionné dans leurs manuels de rédaction et autres documents professionnels, le seul parti pris que les journalistes colombiens s'autorisent dans le cadre de leur travail d'information au sujet du conflit armé interne est en faveur de victimes civiles, car, à leur avis, ce sont elles qui souffrent le plus (Rincón, Ruíz, 2002). La question se pose au moment où cette position partisane de la part des journalistes rend service aux desseins d'un des acteurs armés en accusant implicitement l'adversaire responsable de la mort de ces victimes (Charaudeau et al., 200| : |50-151). D'où l'intérêt des acteurs armés à rendre visible les morts civils causés par l'adversaire (Arboit, 2003 : 831-832 ; Mercier, 2004). À titre d'exemple, la mort en captivité des députés constituait un événement fortement atypique qui se prêtait à un traitement spectaculaire et profitait aux JT dans leur course à l'audience. Ainsi le président de la République, le ministre de la Défense et les représentants des FF.MM. ont-ils saisi toutes les occasions dans lesquelles les jT leur donnaient la parole pour rendre responsables les FARC de ces meurtres ${ }^{23}$. Nonobstant, si la mort des civils

${ }^{23}$ Dans leur communiqué de presse, la guérilla accusait un « groupe militaire non identifié ». 
reçoit plus d'attention de la part des journalistes, des différences sont à relever selon le groupe armé qui en est rendu responsable (graphique 2). Lorsque la mort des civils est attribuée auX FF.MM., les expressions utilisées pour la nommer relèvent d'un lexique qui masque la responsabilité des acteurs étatiques : morir (mourir), fallecer (décéder), perecer (périr). En effet, une personne peut mourir sans forcément être tuée. Les deux tiers de ces expressions, classées dans la catégorie «la mort », sont directement assumés par les journalistes qui ne citent aucune source. De plus, lorsque les JT mentionnent les cas dans lesquels des militaires sont accusés d'avoir tué des civils, il s'agit essentiellement de morts d'une période passée pour lesquels une procédure judiciaire est en cours. Autrement dit, les JT ne mentionnent ces crimes que lorsqu'ils sont pris en charge par la justice. Néanmoins, si l'on considère l'affaire des « faux positifs $»^{24}$, il est probable qu'il y ait plus de civils tués par l'armée et la police colombiennes que ce que les informations télévisées rapportent. En tant que sources ayant plus d'accès aux médias d'information, les FF.MM. masquent le nombre de morts non combattants qu'elles produisent et les journalistes contribuent à minimiser la responsabilité des policiers et militaires en se référant à ces morts comme des « décès », plutôt que comme des « assassinats » ou des « homicides ».

Graphique 2 : Comment la mort des civils est-elle désignée selon l'acteur armé accusé ?25

${ }^{24}$ L'affaire des « faux positifs 》 a éclaté en octobre 2008. Elle concerne l'assassinat de civils, généralement de jeunes hommes provenant des couches sociales défavorisées, par les membres des forces armées de l'État qui déguisent les cadavres en guérilléros pour montrer des résultats positifs dans la lutte contre le «terrorisme ». L'armée récompense ces « bons » résultats par des jours de congé ou des bonifications salariales. Depuis que le scandale a été mis à jour, des militaires ont été licenciés et/ou font l'objet d'enquêtes judiciaires. Le premier rapport du ministère de la Défense à ce sujet, rendu public le 16 octobre 2009, affirmait que depuis 2002 il y avait eu 188 I victimes. En 2010, 194 militaires avaient déjà été traduits en justice et pour d'autres, des procédures d'enquête sont encore en cours. Toutefois, l'ancien président de la République (Á. Uribe) a affirmé qu'il s'agissait de fausses accusations proférées pour empêcher les forces armées de l'État de bien mener leur travail pour combattre « le terrorisme ».

Accès : http://web.presidencia.gov.co/sp/2009/marzo/23/04232009.html, consulté le I4//0/09.

${ }_{25}$ Ce graphique et ceux qui suivent représentent la proportion de désignations classée dans chacune des catégories présentées dans le tableau I, selon l'acteur armé responsable de la mort. 
En revanche, les victimes civiles dont la mort est attribuée, par les sources officielles, aux guérillas sont plus nombreuses dans les informations télévisées et traitées de manière plus spectaculaire, non seulement par la façon de nommer leur mort, mais également à travers les images qui accompagnent les commentaires. Dans un tiers des cas (graphique 2), la mort des civils est désignée avec des expressions qui réprouvent leur mort et mettent en exergue l'atteinte au droit à la vie : 《 assassiner », « massacrer », « cribler de balles », « exterminer ». Seul un quart de ces désignations est énoncé directement par les sources; des trois quarts qui sont relatées par les journalistes, un peu plus de la moitié correspond à des désignations que les journalistes s'approprient. En d'autres termes, ils blâment la mort des civils sans rendre compte du fait que les acteurs armés en parlent à des fins stratégiques. Par le biais des images, les JT renforcent la composante émotionnelle de leurs récits d'information en montrant les funérailles de ces victimes et la souffrance des proches qui pleurent leur absence (photogrammes I à 3). En revanche, l'affliction des proches des combattants morts n'est jamais montrée aux téléspectateurs. Par ailleurs, les JT « donnent à voir 》 les victimes civiles dans leur cercueil, ce qui permet de les « montrer » de manière plus rassurante et surtout moins choquante pour le téléspectateur (Antoine, 1993 : 50). Ce traitement plus « humain » de l'information donné par les JT aux civils, en opposition à celui des combattants, conforte l'argument des journalistes selon lequel les civils représentent les victimes qui souffrent le plus dans ce conflit et qui de ce fait, méritent plus de considération.

$\begin{array}{ccc}\text { Photogramme I } & \text { Photogramme 2 } & \text { Photogramme 3 } \\ \text { Noticias Caracol } & \text { CM\& } & \text { Noticias RCN } \\ \text { 25.06.07 } & 25.06 .07 & 25.06 .07\end{array}$

Si la mort des civils attribuée aux guérillas est surmédiatisée (comme le cas des députés), celle imputée aux paramilitaires est minimisée du fait qu'elle est mentionnée exclusivement dans un cadre judiciaire, lorsqu'elle est jugée et sanctionnée, et non pas lorsqu'elle se produit. Ceci pourrait s'expliquer en partie par l'actualité et non pas uniquement par des choix éditoriaux. En effet, dans la période au cours de laquelle le corpus a été sélectionné, les ex-paramilitaires 
commençaient à rendre leurs 《versions libres $»^{26}$ après leurs démobilisations massives afin d'avoir droit aux privilèges juridiques ${ }^{27}$ et économiques ${ }^{28}$. Les morts que les ex-paramilitaires reconnaissent dans ces auditions sont rapportés par les JT de manière groupée, anonyme et quantifiée : « Le chef paramilitaire, nommé Don Antonio, a reconnu qu'il est responsable d'un demi-million d'assassinats perpétrés par ses hommes $\gg^{29}$. Dans cet exemple, les téléspectateurs ne sont pas informés à propos de ces 500000 personnes, ni sur les conditions de leur mort. Les images diffusées dans les informations traitant des aveux des paramilitaires démobilisés ne correspondent pas non plus aux funérailles ou autres rites dans lesquels la souffrance des proches est exhibée. Les images se rapportent à l'exhumation des fosses communes dont les ex-paramilitaires indiquent l'emplacement exact pour récupérer les dépouilles de leurs victimes (photogrammes 4 à 7 ).

$\begin{array}{cccc}\text { Photogramme 4 } & \text { Photogramme 5 } & \text { Photogramme 6 } & \text { Photogramme 7 } \\ \text { CM\& } & \text { Noticias Caracol } & \text { Noticias RCN } & \text { Noticias Caracol } \\ \text { 12.12.07 } & \text { I5.12.06 } & 13.12 .07 & 29.06 .07\end{array}$

Outre cette manière massifiée et anonyme employée dans les informations télévisées pour se référer aux victimes civiles des paramilitaires, la mort des civils est principalement désignée avec des termes qui expriment la réprobation (《 assassinat », « crime ») ainsi qu'avec des expressions qui minimisent la responsabilité des groupes paraétatiques : «mort », « décès » (graphique 2). Dans ces deux cas, on constate que la proportion de désignations énoncées par les sources est inférieure à $10 \%$, alors que les désignations que les journalistes assument correspondent à la moitié. Lorsque la mort des civils

${ }^{26}$ Version libre ou audition dans laquelle les ex-combattants avouent volontairement leurs crimes auprès de la justice.

27 Les paramilitaires démobilisés n'iront pas plus de 8 ans en prison pour les massacres commis, les disparitions forcées et le déplacement forcé des populations. En revanche, d'autres crimes comme les enlèvements (dont la guérilla est accusée d'être l'un des principaux acteurs) sont punis avec des peines allant de 25 à 40 ans de prison.

28 Participation dans des programmes de réinsertion, de formation, etc.

29 El jefe paramilitar alias Don Antonio se hizo responsable de medio millón de asesinatos perpetrados en la costa atlántica por los hombres bajo su mando (cM\&, 29/06/07). Cet exemple, ainsi que ceux qui suivent, sont traduits de l'espagnol par nous-même. 
causée par les paramilitaires est minimisée à travers des expressions classées dans la catégorie 'la mort', les journalistes citent leurs sources dans les deux tiers des cas. En ce sens, la manière de condamner les paramilitaires qui tuent des civils diffère de celle qui fustige l'action des guérillas. Pour évoquer la mort des victimes civiles des paramilitaires, les JT n'utilisent pas des expressions telles que 《 cribler de balles », 《 exterminer » ou « égorger », alors que des occurrences de ces syntagmes ont été repérées dans les cas où les guérillas sont présumées coupables de la mort des civils. Les morts massives dont les paramilitaires sont responsables ne reçoivent pas la même attention des médias. Dans ce dernier cas, ce qui importe est le nombre de crimes admis. En effet, pour les autorités colombiennes, le fait que les paramilitaires aient rendu les armes et avouent leurs crimes justifie les privilèges juridiques dont ils peuvent être bénéficiaires.

Cette minimisation de la mort causée par les paramilitaires est d'autant plus intéressante de la part des médias que les massacres dont ils sont les auteurs comportent des éléments qui normalement intéressent les médias de masse, car ils sont censés attirer davantage l'attention de récepteurs. Par exemple, une information comme celle diffusée par Noticias Caracol le I I décembre 2007 à propos des aveux de trois ex-paramilitaires illustre ce traitement différencié que le jT donne aux guérillas et aux paramilitaires. L'information diffusée sous la forme d'une brève se limite à rapporter le nombre de crimes avoués par chaque ex-combattant. Aucun détail, aucune information complémentaire sur l'identité des victimes ou sur les mobiles de ces meurtres n'ont été donnés, si ce n'est un complément qui est évoqué juste à la fin par le présentateur en voix off : « John Jairo Esquivel, surnommé le tigre, a reconnu qu'il avait ordonné cinq massacres, plus la mort de sept fonctionnaires de la Fiscalía ${ }^{30}$. Et vous imaginez, [...] le pire c'est que dans certains cas il a dû dépecer ses victimes pour se débarrasser de leurs dépouilles $\gg^{31}$. Tout de suite après, le JT a enchaîné sur un autre sujet qui traitait de la gestion des déchets dans une région du centre du pays. De cette façon, ce dernier détail sur la manière cruelle de procéder des groupes paramilitaires passe presque inaperçu du fait qu'il est formulé comme une sorte de commentaire supplémentaire ; d'autre part, en parlant de « devoir » (« il a dû dépecer... »), la responsabilité du paramilitaire est également atténuée suggérant ainsi qu'il s'agissait pour lui d'une contrainte ${ }^{32}$. Les images montrées dans cette information ne comportent aucun degré de violence. Elles correspondent à celles prises

\footnotetext{
30 La Fiscalía est le bureau du procureur.

31 En Medellín Carlos Mario Jiménez, alias Macaco, reconoció 66 homicidios más [...]. Y en Barranquilla, John Jairo Esquivel, alias el tigre dijo que ordenó 5 masacres y la muerte de 7 funcionarios de la fiscalía en el Cesar.Y qué tal esto, [...] en algunos casos tuvo que descuartizar a sus víctimas para deshacerse de sus cuerpos (Noticias Caracol, I I/I2/07).

32 D'ailleurs, une des raisons pour lesquelles l'opposition critique la façon dont le processus de démobilisation des paramilitaires a été menée concerne cette stratégie des ex-combattants qui n'est pas exclusive au cas colombien et qui consiste à avouer certains crimes, tout en expliquant qu'ils ne faisaient que suivre des ordres, et que pour cette raison, ils ne sont aucunement responsables du déplacement forcé des populations, ni des massacres commis.
} 
lors des auditions et montrent des civils (avocat, juge, ex-paramilitaire) dans une démarche juridique (photogrammes 8 à 10). Pour expliquer cette apparente complaisance et connivence des professionnels de l'information à l'égard des paramilitaires, deux hypothèses pourraient être avancées. En premier lieu, le processus de démobilisation des groupes paraétatiques et les procès judiciaires ont été organisés par les autorités colombiennes (gouvernement de l'ex-président Álvaro Uribe). Comme cela a été mentionné, les propriétaires des grands médias de masse sont proches du gouvernement et de certaines institutions étatiques. Ils peuvent ainsi demander aux journalistes de diffuser des informations qui soutiennent les programmes gouvernementaux. Par ailleurs, de manière à ne pas compromettre leur carrière professionnelle, certains journalistes n'osent pas critiquer le gouvernement de manière explicite. En second lieu, les JT relaient davantage les discours des sources officielles. Ces dernières qui représentent le gouvernement veulent justifier le processus de démobilisation et cela dépit de fortes critiques liées à des problèmes juridico-judiciaires ${ }^{33}$ et sociaux $x^{34}$.

Photogramme 8

Noticias Caracol

। 1.12 .07
Photogramme 9

Noticias Caracol

II.12.07
Photogramme 10

Noticias Caracol

| 1.12 .07

\section{La mort des combattants}

\section{La mort des guérilléros dans les informations télévisées}

Sachant que les acteurs armés et non armés qui représentent l'État sont sollicités par les journalistes en tant que sources davantage que les membres des groupes armés hors-la-loi, il n'est pas étonnant de constater que la moitié des 20 unités d'information dans lesquelles la mort des guérilléros est citée correspond à l'annonce des victoires militaires que les FF.MM. revendiquent. Autrement dit, la mort au combat de ces guérilléros est présentée comme un résultat positif

\footnotetext{
33 Les problèmes juridico-judiciaires concernent les difficultés à assurer les procès des milliers d'excombattants (plus de 30.000 selon le gouvernement), les moyens que cela demande à l'État et les risques d'impunité que cela engendre.

34 Par exemple, parmi les problèmes sociaux, comment et où accueillir ces ex-combattants.
} 
du travail mené par l'armée et la police. Cependant, la responsabilité de ces agents étatiques dans la mort des membres des groupes contre-étatiques n'est clairement affirmée que dans deux informations avec des énoncés comme dans l'exemple suivant: «Dans l'opération Tornado, l'armée, a abattu le chef des milices de la colonne mobile Mariscal Sucre [FARC] $\rangle^{35}$. Le plus souvent (I I unités d'information sur 20), cette responsabilité est suggérée ou énoncée au conditionnel : « 30 guérilléros des FARC seraient morts dans deux opérations avec les forces militaires $\rangle^{36}$. Déclarer que les combattants « sont morts 》 dans les opérations militaires n'implique pas le même sens que si l'on affirme que les forces militaires « ont tué/liquidé/abattu » un autre combattant. Dans d'autres cas, l'utilisation de la voix passive contribue également à atténuer la responsabilité, car l'agent qui cause la mort n'est pas mentionné : « Le 17 juin [...] un terroriste des FARC a été abattu $»^{37}$, « sept guérilléros des FARC ont été abattus lors des combats avec l'armée $»^{38}$.

Outre le fait d'atténuer la violence exercée par l'État, les jT la légitiment, puisque la mort est désignée en utilisant les expressions « dar de baja » (liquider) et « abatir » (abattre). Dans ces cas, les journalistes prennent de la distance à l'égard du lexique employé par les sources, en s'abstenant d'énoncer ces désignations qui déshumanisent les guérilléros morts. En effet, ils n'assument ces expressions que dans $15 \%$ de cas recensés. Les $85 \%$ des expressions classées dans la catégorie «la mort déshumanisée » sont attribuées aux sources en discours rapporté direct (54\%) ou indirect (31 \%). Effectivement, dans le cadre des opérations psychologiques mises en place par les acteurs armés étatiques, ce vocabulaire a pour dessein de déshumaniser l'adversaire auprès de leurs troupes et de la population civile pour ainsi justifier le fait de le tuer. Dans aucun cas, la mort des guérilléros n'est désignée avec les expressions qui désapprouvent leur mort en mettant l'accent sur le caractère violent de leur mort : assassinat, crime ou massacre (graphique 3), comme c'est le cas pour les militaires et les paramilitaires. Si l'on considère les décès des membres des FF.MM, les guérilléros décédés sont moins souvent désignés comme corps, cadavres ou dépouilles. C'est, en revanche, leur condition de hors-la-loi qui est mise en avant. D'un autre côté, lorsque la mort des guérilléros survenue dans le passé est rappelée par les JT, il s'agit principalement des informations relatives à la mort des députés. En fait, les sources officielles affirmaient que la mort des politiciens était la conséquence de représailles de la guérilla pour venger leurs camarades tués. Cet argument permet de montrer l'adversaire comme un être vindicatif, rancunier qui prend sa revanche avec des civils sans défense.

\footnotetext{
35 El ejército en la operación tornado dio de baja al jefe de las milicias de la columna móvil Mariscal Sucre (Telepaís, I I/12/06).

3630 guerrilleros de las FARC habrían muerto en 2 operaciones de las fuerzas militares (Noticias RCN, 26/06/07)

37 El 17 de junio [...], fue dado de baja un terrorista de las FARC (Noticias RCN, 28/06/07).

38 Siete guerrilleros de las FaRC fueron abatidos en combate con el ejército (Noticias RCN, I3/I2/07).
} 
Au sein du groupe de combattants, seuls les cadavres des guérilléros « abattus » sont « montrés » aux téléspectateurs (photogrammes || à |3). D'ailleurs, les images de cadavres ne sont présentes que dans 13 unités d'information sur les 187 analysées : 5 d'entre elles correspondent à des civils et 8 à des combattants contre-étatiques. Dans la plupart des cas, ces personnes décédées apparaissent couvertes: dans des housses noires, sous un drap blanc ou dans un cercueil (photogrammes I à 3 et I| à |3). Quant à la mort provoquée par les paramilitaires, ce sont des ossements déterrés qui sont montrés (photogrammes 4 à 7). Une seule information montre aux téléspectateurs un cadavre qui n'est pas dissimulé (photogramme 14) ; il s'agit d'une image d'archive de Raúl Reyes, porte-parole de la guérilla des FARC « abattu » par l'armée colombienne en mars 2008 dans une opération militaire très controversée sur le sol équatorien ${ }^{39}$. À l'occasion du premier anniversaire de la mort en captivité des I I députés, le ministre de la Défense a annoncé que l'ordinateur de Raúl Reyes contenait des informations selon lesquelles les FARC préparaient un plan pour montrer que la mort des députés résultait d'une erreur des FF.MM. En parlant de cette annonce du ministre, le jT Telepaís a diffusé une image d'archive du guérilléro mort. C'est la seule et unique image dans laquelle un cadavre est montré découvert. Cette différence s'explique par le fait que l'opération militaire de l'armée colombienne dans laquelle le porte-parole des FARC a été tué est revendiquée comme la plus importante victoire militaire remportée contre cette guérilla. En cela, ce corps constitue un trophée de guerre que les acteurs étatiques, à l'aide des médias de masse, s'empressent de vanter ${ }^{40}$.

Enfin, ces quelques images de personnes décédées ou de leurs ossements n'utilisent pas de cadres serrés. Les plans larges dominent : le plan de demiensemble (utilisé dans 10 unités d'information), ainsi que le plan général et le plan moyen ( 6 unités d'information pour chacun). Ces corps dissimulés confirment la volonté des médias colombiens de ne pas «trop » montrer d'images violentes en relation avec le conflit armé. La dissimulation de ces corps pourrait également s'expliquer par des raisons culturelles, comme le tabou autour de la mort présent en Occident ou comme une preuve de respect de la part des médias à l'égard des proches.

\footnotetext{
39 Cette opération a engendré une crise diplomatique, qui n'est pas complètement résolue, entre les deux pays. La Colombie défend son intervention en disant que dans la lutte contre le terrorisme, il faut la collaboration de tous les pays. L'Équateur dénonce une violation de sa souveraineté.

${ }^{40}$ Le même type de couverture médiatique a été constaté en septembre 2010, lorsque les FF.MM. ont tué le chef militaire des FARC, surnommé Mono Jojoy. Des images en couleur de la dépouille ensanglantée de ce guérilléro ont été largement diffusées par les médias.
} 


$\begin{array}{cccc}\text { Photogramme I } & \text { Photogramme 12 } & \text { Photogramme 13 } & \text { Photogramme I4 } \\ \text { Noticias RCN } & \text { Telepais } & \text { Noticias RCN } & \text { Telepais } \\ \text { 13.12.07 } & \text { I2.06.06 } & \text { I4.06.06 } & \end{array}$

En résumé, les sources officielles rapportent la mort de leurs adversaires essentiellement pour montrer leur défaite et, en même temps, justifier les opérations militaires à leur encontre. La violence exercée par l'État est ainsi présentée comme ciblée, puisqu'elle ne s'applique qu'aux groupes hors-la-loi dont les sources officielles s'empressent d'affirmer qu'ils n'ont pas de revendications légitimes. Relevons enfin qu'aucun guérilléro mort, mentionné dans le corpus, n'est attribué aux groupes paramilitaires, alors que dans le discours de ces derniers, ils apparaissaient comme leurs principaux adversaires. Dans une seule unité d'information, traitant des aveux des ex-paramilitaires, un journaliste mentionne qu'un démobilisé avait reconnu : « Parmi les personnes assassinées, on trouve un conseiller municipal, un brigadier de l'armée et un arbitre de football accusés d'être des collaborateurs de la guérilla par les [paramilitaires], mais ils ont été tués par erreur $\gg^{41}$.

Graphique 3 : Comment la mort des guérilléros est-elle désignée selon l'acteur armé accusé ?

\footnotetext{
${ }^{41}$ Entre los asesinados se encuentran un concejal del municipio de Segovia, un cabo segundo del ejército y un árbitro de fútbol a quienes las autodefensas señalaban de ser colaboradores de la guerrilla pero que mataron por error (Noticias Caracol, I0/I2/07).
} 


\section{La mort des acteurs armés qui représentent l'État}

Sur les 187 unités d'information analysées, 16 mentionnent la mort des membres des FF.Mm. Néanmoins, il s'agit essentiellement des militaires et des policiers morts dans le passé. Dans aucune information les JT n'annoncent leur mort au combat. En effet, il n'est pas convenable de parler de ses propres morts, car cela peut démoraliser les troupes. Ne pas montrer ses morts correspond à une stratégie des armées consistant à limiter l'image de la défaite (Mercier, 2004 : I 5 I). Dans cette même logique, aucune information ne montre des cadavres de militaires ou de policiers. Les armées n'exhibent ou ne parlent de leurs morts que si cela répond à une stratégie précise, comme celle de mobiliser la population contre l'adversaire. Cette démarche a pour finalité de justifier l'augmentation du budget consacré à la guerre, le renforcement des opérations militaires et/ou l'augmentation des pouvoirs accordés à l'armée. Pour illustrer cette instrumentalisation de la mort, citons un reportage diffusé par CM\& le 17 juin 2008. Ce jour-là, le présentateur annonçait que « les forces militaires ont révélé que $|5|$ soldats sont morts dans des opérations contre la guérilla et le narcotrafic $»^{42}$. Ensuite, la journaliste poursuivait son commentaire en énumérant le nombre de soldats blessés et morts à cause des mines antipersonnel ou au combat. Le téléspectateur pouvait donc comprendre que le sujet traité par l'information était la mort des militaires. Néanmoins, la dernière phrase énoncée par la journaliste suggérait que ce bilan des militaires morts dans le conflit était une manière de justifier une demande des FF.MM. au législatif colombien lorsqu'elle concluait que « le commandant des forces militaires s'est exprimé lors d'un débat au Congrès pour demander des pouvoirs extraordinaires pour le président de la République $\gg^{43}$. Aucune précision n'a été donnée aux téléspectateurs à propos du type de pouvoir extraordinaire demandé. II s'agit seulement de rappeler le nombre de soldats morts dans le conflit pour argumenter une demande afin d'accroître la marge de manœuvre légale des militaires sur le terrain.

Parler de la mort des membres des FF.MM. est aussi une tactique utilisée pour montrer à la population que les forces armées sont « épurées 》 de mauvais éléments, car les militaires accusés de la mort d'autres militaires sont jugés. Ceci permet également de justifier la capture ou la mort des guérilléros qui, à leur tour, avaient tué des militaires. Dans les trois-quarts des cas, la responsabilité des guérilléros, des paramilitaires et des militaires, selon le cas, est explicite et énoncée à l'indicatif : « un juge a refusé la liberté des militaires accusés du massacre de 10 policiers et un civil à Jamundí,Valle $\rangle^{44}$. En revanche, dans un quart des informations concernées, cette responsabilité est uniquement supposée :

42 Las fuerzas militares revelaron que 151 soldados han muerto durante lo corrido de este año en los operativos que se adelantan contra la guerrilla y el narcotráfico.

43 El comandante de las Fuerzas Militares se pronunció al término de un debate en el congreso en el cual se piden facultades extraordinarias para el presidente de la república.

${ }^{44}$ Un juez [...] negó la libertad a los militares implicados en la masacre de 10 policías y un civil en Jamundí, Valle (Noticias RCN, I3/I2/06). 
« Une opération du DAs et de la Fiscalía a permis l'arrestation des 26 membres présumés des FARC et de l'EPL accusés du massacre de 12 détectives du DAS et de soldats de l'armée $\gg^{45}$, suggérant aux téléspectateurs que l'appartenance de personnes arrêtées aux guérillas n'est pas confirmée.

Les deux exemples qui viennent d'être cités montrent également que la mort des combattants étatiques (graphique 4) n’est pas désignée de la même manière que celle des combattants contre-étatiques (graphique 3) et paraétatiques (graphique 5). Plus concrètement, si la guérilla est accusée, la mort des militaires est alors commentée avec des expressions comme « massacre », « assassinat 》 ou « crime »: «Des crimes comme l'assassinat de 29 membres de l'armée sont attribués à cette guérilla $»^{46}$, « Ce policier a été égorgé [par les FARC] $»^{47}$. Les quatre cinquièmes de ces désignations de réprobation sont énoncés par les journalistes. Dans la moitié des cas, ils ne rapportent pas les propos des sources, mais prennent en charge ce lexique négatif. Ces expressions sont aussi utilisées pour nommer la mort des civils, victimes innocentes du conflit. En se présentant comme des « victimes », les FF.MM. délégitiment leur adversaire. En revanche, si d'autres militaires sont incriminés, les JT parlent de « l'affaire » pour indiquer que ces cas sont soumis à la justice. Les désignations correspondant à la catégorie 《la mort affaire $»^{48}$ sont toujours assumées par les journalistes. Enfin, lorsque les paramilitaires sont rendus responsables, la mort des militaires est présentée par les sources officielles (leurs propos sont, dans tous les cas, rapportés par les journalistes) comme un « décès » ou une «mort », ce qui masque la violence avec laquelle ces morts se sont produites. En d'autres termes, la mort des combattants étatiques reçoit un traitement plus « humain » et compatissant de la part des JT, proche de celui qui est accordé aux victimes civiles. De cette façon, les autorités cherchent à gagner le soutien de la population civile dans leur lutte contre la guérilla.

\footnotetext{
45 Una operación del DAS y la fiscalía permitió la captura de 26 presuntos miembros de las FARC y del EPL sindicados de participar en la masacre de 12 detectives del das y soldados del ejército (CM\&, 14/12/06).

${ }^{46}$ A [esta guerrilla] se le atribuyen crímenes como el asesinato de 29 miembros del ejército (Telepaís, I |/|2/07).

47 A ese policía lo degollaron (Noticias Caracol, 28/6/07).

${ }^{48}$ La définition de cette catégorie est donnée dans le tableau I.
} 
Graphique 4 : Comment la mort des militaires est-elle désignée selon l'acteur armé accusé ?

\section{La mort des paramilitaires dans les informations télévisées}

Après les dernières démobilisations massives de paramilitaires qui ont eu lieu en juin 2006, le gouvernement colombien affirme avoir démobilisé plus de 30000 paramilitaires. II assure qu'il n'y a plus de groupes paraétatiques, ce qui explique en partie le nombre réduit d'informations télévisées faisant référence à la mort de ces combattants. La majorité des informations traitant des paramilitaires concerne les auditions dans lesquelles ils reconnaissent leurs crimes. En effet, il n'y a que trois informations qui mentionnent un paramilitaire mort. Elles annoncent l'émission du mandat d'arrêt émis contre un paramilitaire fugitif pour avoir ordonné la mort de son frère qui était le chef de la confédération des groupes paramilitaires créée en 1997, AUc (Autodéfenses unies de Colombie). Aucune information ne fait mention de la mort des paramilitaires attribuée aux FF.MM. ou aux guérilléros, car il s'agirait de morts au combat, ce qui contredirait la version officielle, selon laquelle les paramilitaires n'existent plus. Dans ces trois informations, les expressions qui désignent la mort s'inscrivent dans un registre juridico-judiciaire (« homicide », « homicide aggravé ») et de réprobation (« assassinat », « crime »). Ces expressions sont prises en charge par les journalistes dans les trois quarts des cas et par les sources officielles dans un quart des cas. Ici, l'objectif est de montrer que les paramilitaires, après leur démobilisation, sont toujours sous contrôle et que celui qui ne tient pas ses engagements de retourner à la vie civile ne bénéficie pas des privilèges accordés par le gouvernement. Par opposition aux cas concernant la mort des combattants étatiques et contre-étatiques, les désignations autour du mot « mort/la mort » sont moins fréquentes (graphique 5). 
Graphique 5 : Comment la mort des paramilitaires est-elle désignée selon l'acteur armé accusé ?

\section{Conclusion}

La gestion de la mort intrinsèque aux confrontations armées dans les médias de masse répond aux objectifs de légitimation, de témoignage, de mobilisation de certains secteurs de la population ou de construction d'une mémoire collective, comme le montre l'ensemble des articles consacrés ici à la mort. Plus concrètement, la monstration de la mort et des morts ou leur dissimulation peuvent être utilisées à des fins stratégiques par les acteurs en conflit, notamment par ceux qui ont plus d'accès aux médias. En ce sens, les réflexions avancées par d'autres chercheurs (Le Guay, 2008), à propos de la banalisation de la mort par les médias, méritent d'être replacées dans le contexte du traitement médiatique des conflits internes par les médias d'information nationaux. Dans le cadre du conflit armé en Colombie, les résultats de cette analyse font apparaître que si les informations télévisées parlent fréquemment de la mort (42 \% du corpus), notamment des civils, la mort demeure peu visible à l'écran, puisque seulement $3 \%$ des unités d'information analysées montrent des personnes décédées. Le lexique utilisé pour parler de la mort liée au conflit rentre dans une stratégie de justification des actions armées pour combattre l'ennemi. La mort des civils est instrumentalisée afin de mobiliser la population contre les groupes armés déclarés seuls responsables de leur mort. La mort des combattants est traitée de manière différenciée par les JT nationaux, dans la mesure où les sources officielles font plus souvent état des morts du camp adverse que de leur propre camp. Dans ce cas, la stratégie consiste à vouloir faire prendre conscience à l'adversaire de l'ampleur de sa défaite et à convaincre la population civile que la victoire militaire est imminente. Plus spécifiquement, la mort des combattants étatiques est présentée comme s'il n'était pas question des combattants qui meurent au combat et qui donnent aussi la mort. De plus, la violence des agents étatiques est justifiée. En réalité, l'accent mis par les JT sur les morts des civils causées 
par les guérillas plutôt que par les FF.MM. sert à démontrer que les principaux adversaires de l'État ne méritent aucune reconnaissance d'ordre politique. Pour leur part, les paramilitaires sont présentés comme uniquement responsables de morts commises dans le passé. Quant aux civils, ils sont présentés comme les victimes à travers lesquelles, les JT peuvent prendre position vis-à-vis du conflit. Par ce biais, ils tentent d'impliquer les téléspectateurs sans prendre pour autant le risque d'être critiqués par manque d'impartialité. D'où l'intérêt de distinguer les expressions utilisées pour désigner la mort selon les enjeux de (dé)légitimation qu'elles comportent. En conséquence et même si les journalistes revendiquent l'objectivité de leur travail, la relation que les acteurs armés entretiennent avec les professionnels de l'information est déterminée par des objectifs militaires. Néanmoins, les professionnels de l'information ne sont pas toujours en mesure de répondre aux pressions des sources et les citoyens ne sont pas forcément conscients de cette instrumentalisation des médias par les acteurs en conflit. En fait, les principes qui définissent ce que les professionnels appellent l'objectivité de l'information (Chalaby, 1998) sont incompatibles avec la stratégie militaire des combattants. « Pris en otage » ou en raison de leurs propres perceptions sur leur pays et sur la situation de violence, les journalistes finissent par reproduire le discours des sources officielles, notamment les catégories de désignation imposées par les acteurs armés dans une logique de guerre. Ce ne sont pas seulement les FF.MM. qui parlent des guérilléros « abattus » ou des policiers « massacrés » au combat, mais aussi les journalistes. Pour cette raison, la question de la responsabilité des médias doit être soulevée, non seulement parce qu'à travers leurs discours d'information ils proposent une certaine lecture du conflit armé, mais surtout parce que les médias participent au processus par lequel certains éléments peuvent se charger d'une valeur testimoniale (Walter, 2005). Aussi les médias colombiens ont-ils choisi d'assumer leur responsabilité en ne diffusant pas d'images violentes qu'ils pourraient considérer inutiles, l'occultation de la « réalité »du conflit qui en résulte peut empêcher les Colombien-ne-s de prendre conscience de cette réalité. Comme le soulignait le journaliste colombien Hollman Morris, lors d'un entretien en novembre 2008, « un massacre qui n'est pas montré [par les médias] est un massacre qui n'a pas existé [pour les citoyens] ». Effectivement, désigner et montrer certains aspects de la réalité constitue une forme de pouvoir des médias qui consiste, non seulement à faire exister ce qui est nommé et montré, mais aussi à en proposer une certaine lecture selon les désignations et les images retenues (Charaudeau, 2005). Ce qui est montré est mis en exergue, alors que ce qui ne l'est pas est minoré, voire occulté ou nié (Thanassekos, 2008). La mort comme caractéristique inhérente à la guerre, en étant voilée et instrumentalisée en faveur des desseins d'un des acteurs du conflit, pose d'une part la question de la mémoire que les médias contribuent à construire sur ce conflit armé par le biais de l'occultation d'une partie de la « réalité »; et d'autre part, elle soulève la question de la légitimation de la violence menée par l'un des belligérants. 


\section{Références}

Antoine F., 1993, « Mourir au JT. Les cadavres exquis de l'information télévisée », Annuaire français de relations internationales, 4, pp. 45-64.

Arboit G., 2006, « À nos morts, médiatiser la mort au champ d'honneur : un enjeu mémoriel et politique », Quaderni, 62(1), pp. 81-92.

Bardin L., 1977, L'analyse de contenu, Paris, Presses universitaires de France, 200I.

Bertherat B., 2003, « Cadavre à la "une". La télévision et la mort de Jacques Mesrine, ennemi public n I (1979) », Le Temps des médias, I ( ), pp. I 19- I 38.

Bonilla J., Patiño G., eds, 200I, Comunicación y Política. Viejos conflictos, nuevos desafíos, Bogotá, Centro Editorial Javeriano.

Bushnell D., 1993, The Making of Modern Colombia. A nation in Spite of Itself, Berkeley, University of California Press.

Chalaby J., 1998, The Invention of Journalism, Houndmills, Basingstoke, Hampshire and London, MacMillan Press LTD.

Charaudeau P., 2005, Les médias et l'information. L'impossible transparence du discours, Bruxelles, De Boeck Université.

Charaudeau P., Lochard G., Soulages J.-C., Fernandez M., Croll A., 200I, La télévision et la guerre. Déformation ou construction de la réalité ? Le conflit en Bosnie (1990-1994), Bruxelles, De Boeck Université.

CNTV, 1997, Servicio Público y Cobertura, Sistema de Televisión en Colombia, Bogotá, cNT.

- 2009, Módulo de Televisión. Estudio General de Medios (egm). Segunda ola 2008, Bogotá, CNT.

Garibay D., 2003, Des armes aux urnes, Processus de paix et réinsertion politique des anciennes guérillas en Colombie et au Salvador, Thèse de doctorat en science politique, Institut d'études politiques de Paris.

Géré F., 1997, La guerre psychologique, Paris, éd. Economica.

Giraldo D., Roldán I., Flórez M., 2003, Periodistas, guerra y terrorismo, Bogotá, Planeta/ Universidad Sergio Arboleda.

Gonzalez O., 20 I0, «Violence homicide en Colombie : déviance ou "débrouille" ? » Sociologos. Revue de l'association française de sociologie, 5, Mis en ligne le 23/07/I0. Accès : http://sociologos.revues.org/2490.

Granada S., Restrepo J., Vargas A., 2009, « El agotamiento de la política de seguridad: Evolución y transformaciones recientes en el conflicto colombiano », pp. 27-124, in : Restrepo J.,Aponte D., éds, Guerra y violencias en Colombia. Herramientas e interpretaciones, Bogotá, Pontificia Universidad Javeriana.

IISS, 2009, The Military Balance, London, Routledge.

Jankélévitch V., 1977, La mort, Paris, Flammarion, 2008.

Le Guay D., 2008, «Représentation actuelle de la mort dans nos sociétés : les différents moyens de l'occulter », Études sur la mort, | 34, pp. I | 5- 123. 
López F., 2005, « Aprendizajes y encrucijadas del periodismo. Entre la paz de Pastrana y la seguridad democrática de Uribe », Palabra Clave, 13, pp. 7-45.

Mercier A., 1993, « Médias et violence durant la guerre du Golfe », Cultures \& Conflits, La violence politique dans les démocraties européennes occidentales, 9/10, pp. 377-388.

— 2004, « Montrer ou non les morts à l'écran », pp. I 5 | - 163, in : Charon J.-M., Mercier A., coords, Armes de communication massive. Informations de guerre en Irak : 1991 -2003, Paris, Éd. CNRS.

MPP, Programa Por La Paz, Universidad Javeriana, 2006, Prensa, conflicto armado y región. Aprendizajes del diplomado Periodismo responsable en el conflicto armado, Bogotá, Corporación Medios para la paz.

Von Münchow P., 2004, Les journaux télévisés en France et en Allemagne. Plaisir de voir ou devoir de s'informer, Paris, Presses Sorbonne Nouvelle.

PAN, 2004, El papel de las fuentes oficiales en la calidad del periodismo colombiano, Centro de Competencia en Comunicación para América Latina, Bogotá. Accès : http://www.c3fes. net/docs/fuentesoficiales.pdf - Consulté le 29/02/08.

Pécaut D., 1994, «Violence et politique : quatre éléments de réflexion à propos de la Colombie », Cultures \& Conflits, Disparitions, 13-14, pp. I55- 166.

- 2003, Midiendo fuerzas. Balance del primer año del gobierno de Álvaro Uribe Vélez, Bogotá, Planeta.

PNUD Colombia, 2003, Informe Nacional de Desarrollo Humano para Colombia 2003. El Conflicto, callejón con salida, Bogotá, El Malpensante.

Rabatel A., Florea M.-L., 20 I I, «Re-présentations de la mort dans les médias d'information », Questions de communication, 19, pp. 7-28.

Rincón O., Ruiz M., éds, 2002, Bajo todos los fuegos. Los periodistas en el conflicto colombiano, Bogotá, Proyecto Antonio Nariño.

ThanassekosY., 2008, « Les processus de qualification, de disqualification et de requalification des lieux de mémoire (1950-2000) », pp. 219-227, in : Fleury B., Walter J., dirs, Qualifier des lieux de détention et de massacre, Nancy, Presses universitaires de Nancy.

Thomas L.-V., 1988, La mort, Paris, Presses universitaires de France, 2003.

Serrano Y., 2010 , Cadrage informatif du conflit armé en Colombie dans les journaux télévisés nationaux : informations médiatiques et communication de guerre, Thèse de doctorat en science de l'information, de la communication et des médias, université de Genève.

Veniard M., 2003, « Guerre dans le discours de presse sur les conflits armés : orientation argumentative et marque de point de vue », Actes des rencontres de l'école doctorale Langage et langues, Paris, pp. 23-26.

Viallon P., 1996, L'analyse du discours de la télévision, Paris, Presses universitaires de France.

Walter J., 2005, La Shoah à l'épreuve de l'image, Paris, Presses universitaires de France. 